\title{
Geometric nonlinear dynamic response of wind turbines with different power performance
}

\author{
Xiaohang Qian ${ }^{1}$, Zhiteng Gao ${ }^{1}$, Zhiyong Zhang ${ }^{2}$, and Tongguang Wang ${ }^{1 *}$ \\ ${ }^{1}$ Jiangsu Key Laboratory of Hi-Tech Research for Wind Turbine Design, Nanjing University of Aeronautics and Astronautics, Nanjing, \\ Jiangsu, 210016, China \\ ${ }^{2}$ Jingyuan Urban Construction Investment Company Limited. Baiyin, Gansu, 730600, China
}

\begin{abstract}
As the size of wind turbine blades increases, the influence of geometric nonlinearity on aerodynamic, structural and design of blades becomes more and more serious. In this work, the efficient aero-elastic calculation of large flexible blades is studied. In order to solve the problem of efficient aeroelastic caculation of large flexible blades, this work applied the geometrically exact beam theory based on Legendre spectral finite element and coupled with the blade element momentum theory to establish the aero-elastic analysis model of large flexible blades. This model can efficiently calculate the deformation and load on the blade under aerodynamic loading and fully consider the influence of geometric nonlinearity caused by deformation on aeroelastic ability. Taking NREL 5MW and IEA 15MW wind turbines as examples, the linear and nonlinear dynamic responses of these two wind turbine blades are calculated. The result shows that the neglect of nonlinear effect will bring error. From $5 \mathrm{MW}$ wind turbine to $15 \mathrm{MW}$ wind turbine, the numerical error increased by $27.88 \%$. The influence of geometric nonlinearity of blades on dynamic responses is analysed, which is of great significance to improve the design level of large-scale wind turbines.
\end{abstract}

\section{Introduction}

With the increase of the size of composite blades, nonclassical effects, such as geometric nonlinearity, warpage in and out of the section, have significant effects on the dynamic response of blade structures. However, analysis of composite-material structures is more difficult than their isotropic counterparts due to elastic-coupling effects ${ }^{[1]}$. The traditional modal superposition method is still suitable for calculating the deformation of low power wind turbines. However, for large-scale wind turbines, large deflections may occur under working conditions. At this time, the traditional linear method can no longer accurately predict the dynamic response of blades. Developing a geometrically nonlinear beam model for the pre-twisted and curved composite blade is a relatively new focus of the wind turbine community $[2]$. The strain in the blade section is usually small strain. Therefore, the geometric nonlinearity is mainly caused by the finite rotation of the blade section. In this work, the geometrically exact beam theory based on the Legendre spectral finite element is applied, which is based on the classical Timoshenko beam theory and takes into account the section rotation. The structure modelling involving the coupled flapwise, edgewise and torsional degrees of freedom ${ }^{[3-4]}$. At present, the commonly used methods in the aerodynamic calculations are the actuator line - large-eddy simulation (AL-LES) method $^{[5-6]}$, blade element momentum theory, and so on. Due to the high computational complexity of the ALLES, the geometrically exact beam theory is coupled with the blade element momentum theory to establish the aeroelastic analysis model of wind turbine blades in this work. This model can accurately calculate the blade deformation under aerodynamic load and fully consider the influence of geometric nonlinearity caused by deformation on aeroelastic stability.

\section{Numerical Methods}

In this work, the numerical calculation of geometrically exact beam theory based on Legendre spectral element method is realized. Geometrically exact beam theory is mainly based on accurate description deformation to achieve geometric nonlinearity. This theory is characterized by the ability of beams subjected to initial bending and torsion to withstand large displacements and rotations. With a proper three-dimensional crosssection analysis, all the coupling effects of six degrees of freedom, including stretching, bending, shearing, torsion, out of plane shear, torsion warping and in plane warping can be covered by geometrically exact beam theory. "Geometrically exact" refers to the absence of approximations between the original geometry and the distorted geometry in the reformulations. As shown in Fig.1.

\footnotetext{
* Corresponding author: tgwang@nuaa.edu.cn
} 
The governing equations of nonlinear motion of geometrically exact beam theory are as follows:

$$
\begin{gathered}
\underline{\dot{h}}-\underline{F^{\prime}}=\underline{f} \\
\dot{g}+\dot{\tilde{u}}-\underline{M^{\prime}}-\left(\widetilde{x_{0}^{\prime}}+\widetilde{u^{\prime}}\right) \underline{F}=\underline{m}
\end{gathered}
$$

where $\underline{h}$ and $g$ are the linear and angular momenta resolved in the inertial coordinate system, respectively, $\underline{F}$ and $\underline{M}$ are the beam's sectional forces and moments, respectively, $\underline{u}$ is the one-dimensional displacement of the reference line, $x_{0}$ is the initial position vector of a point along the beam's reference line, and $\underline{f}$ and $\underline{m}$ are the distributed force and moment applied to the beam. $(\bullet)^{\prime}$ indicates a derivative with respect to the beam axis $\mathrm{x},(\cdot)$ indicates a derivative with respect to time. $\widetilde{(\bullet)}$ defines a second-order, skew-symmetric tensor corresponding to the given vector.

The constitutive equation reveals the relationship between velocity and momentum and between strain and cross-sectional force:

$$
\begin{aligned}
& \left\{\begin{array}{l}
\underline{h} \\
g
\end{array}\right\}=\underline{\underline{M}}\left\{\begin{array}{l}
\underline{u} \\
\underline{\omega}
\end{array}\right\} \\
& \left\{\begin{array}{l}
\underline{F} \\
\underline{M}
\end{array}\right\}=\underline{\underline{K}}\left\{\begin{array}{l}
\underline{\varepsilon} \\
\underline{\kappa}
\end{array}\right\}
\end{aligned}
$$

where $\underline{\underline{M}}$ and $\underline{\underline{K}}$ are the $6 \times 6$ sectional mass and stiffness matrices, and $\underline{\varepsilon}$ and $\underline{\kappa}$ are the one-dimensional strains and curvatures, $\underline{\omega}$ is the angular velocity vector, represented by the rotation tensor $\mathrm{R}$ as:

$$
\underline{\omega}=\operatorname{axial}\left(\underline{\underline{R}} \underline{\underline{R}}^{T}\right)
$$

The second-order tensor $\stackrel{A}{=}$ associated with the axial vector $\underline{a}$ can be expressed as $\underline{a}=\operatorname{axial}(\underline{\underline{A}})$, and its components can be expressed as:

$$
\underline{a}=\operatorname{axial}(\underline{\underline{A}})=\left\{\begin{array}{l}
a_{1} \\
a_{2} \\
a_{3}
\end{array}\right\}=\frac{1}{2}\left\{\begin{array}{l}
A_{32}-A_{23} \\
A_{13}-A_{31} \\
A_{21}-A_{12}
\end{array}\right\}
$$

The one-dimensional strain is expressed as:

$$
\left\{\begin{array}{l}
\underline{\varepsilon} \\
\kappa
\end{array}\right\}=\left\{\begin{array}{c}
x_{0}^{\prime}+\underline{u}^{\prime}-\left(\underline{\underline{R}} \underline{\underline{R}}_{0}\right) \bar{l}_{1} \\
k
\end{array}\right\}
$$

The three equations above, including the equations of motion, constitutive equations and kinematics equations, give a complete mathematical description of the geometrically exact beam theory.

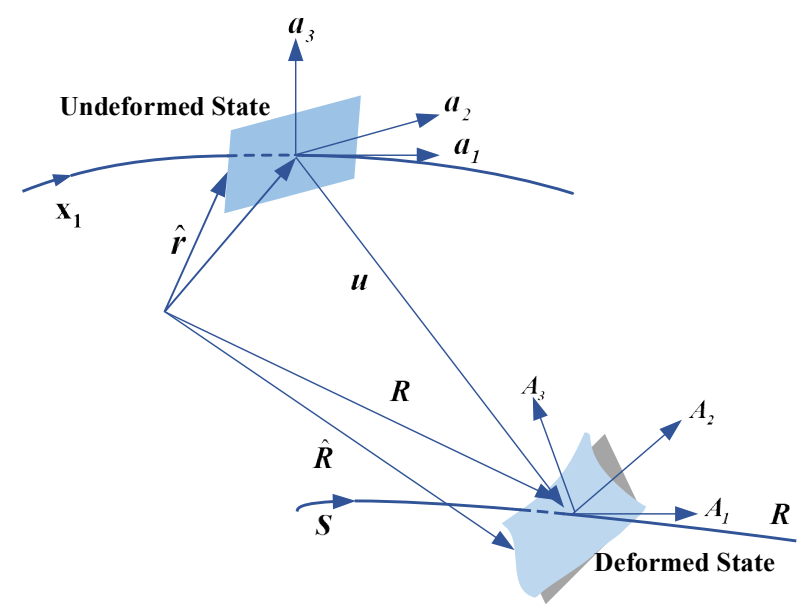

Fig.1. Schematic of the beam in undeformed and deformed states

\section{Results and Discussion}

In this work, two turbine blades of NREL 5MW and IEA $15 \mathrm{MW}$ are simulated and analysed. Among them, the wind turbine diameter of $5 \mathrm{MW}$ blade is $126 \mathrm{~m}$, hub height is $90 \mathrm{~m}$, and the total length of blade is $61.5 \mathrm{~m}$. As for the $15 \mathrm{MW}$ blade, the diameter of the wind turbine is $240 \mathrm{~m}$, the hub height is $150 \mathrm{~m}$, and the total length of the blade is $117 \mathrm{~m}$.

\subsection{Blade model comparison}

As shown in Fig.2, there are some differences in the twist distribution between $5 \mathrm{MW}$ and $15 \mathrm{MW}$ wind turbine blades. The twist distribution of 5MW blade keeps constant near the root of blade, then decreases gradually until the tip of blade; but the twist of $15 \mathrm{MW}$ blade decreases gradually from root to $100 \mathrm{~m}$, and then increases near tip, this indicates some unloading at the tip, which releases some energy production to mitigate the maximum thrust loads at the most flexible part of the blade.
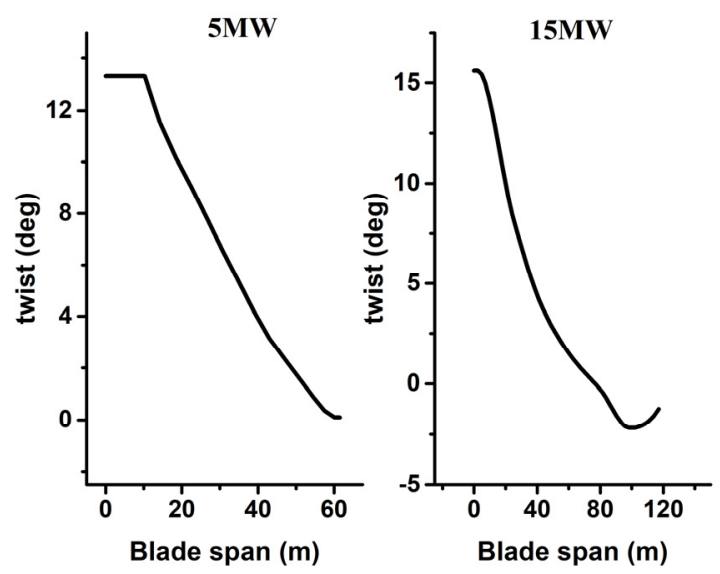

Fig.2. Distribution of the two wind turbine blades twist along blade span 


\subsection{Power Validation}

This paper simulated the electrical power of 5MW and $15 \mathrm{MW}$ wind turbines under the condition of uniform inflow respectively by using the modal superposition method and the geometrically exact beam theory. The power value at a certain wind speed is all calculated by using the average value of the stable operation period of the wind turbine. From Fig.3, linear represents the modal superposition method and nonlinear represents the geometrically exact beam method.

As shown in Fig.3, for the 5MW wind turbine, the rated wind speed is around $11.4 \mathrm{~m} / \mathrm{s}$. the nonlinear result of power is consistent with the linear result basically. While the nonlinear power values are sightly lower than the linear results when the wind speed is lower than the rated wind speed. As for the $15 \mathrm{MW}$ wind turbine, the rated wind speed is around $10.59 \mathrm{~m} / \mathrm{s}$. However, under the nonlinear method, the rated wind speed is around $11.5 \mathrm{~m} / \mathrm{s}$, and the power value of the corresponding wind speed below this wind speed is smaller than that under the linear result, and the difference is larger than that of $5 \mathrm{MW}$ wind turbine. Therefore, considering the torsional deformation of the blade, the power may be reduced. Because the model with torsional deformation has a lower load and lower power generation. It can be observed that the power difference is larger when the wind speed is close to $11.5 \mathrm{~m} / \mathrm{s}$. Such difference indicates that there is no significant difference in the power forecast of the turbine set with low wind speed and small deformation blades by using the modal superposition method and the geometrically exact beam theory. Compared with the modal superposition method, the geometrically exact beam theory takes the torsional deformation of the blade into account in the analysis of the structure, which realizes the bending-torsional coupling of the blade.

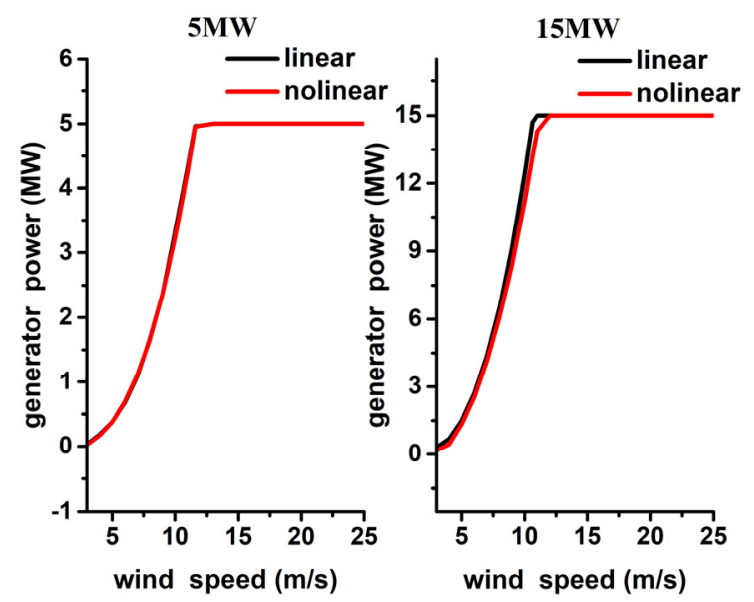

Fig.3. Power performance of wind turbines under different wind speeds

\subsection{Dynamic responses of blade}

For modern wind turbines blades, the curved blade geometry and the use of composite materials will lead to complex flapwise-edgewise and bending-torsion structural coupling effects, which have a great influence on the aeroelastic response, this can lead to large and complex deformation of the blade. The aeroelastic parameters of $5 \mathrm{MW}$ and $15 \mathrm{MW}$ wind turbine blade were calculated under normal operating conditions. The cut-in wind speed of the two wind turbines is $3 \mathrm{~m} / \mathrm{s}$ and the cutout wind speed is $25 \mathrm{~m} / \mathrm{s}$, so the dynamic response of blades with wind speed ranging from 3 to $25 \mathrm{~m} / \mathrm{s}$ is calculated.

As shown in Fig.4, for 5MW wind turbine, the largest flapwise tip deflection occurs under the rated wind speed when the wind turbine reaches the rated electrical power. The tip deflection is $5.51 \mathrm{~m}$. The difference between linear and nonlinear result near the rated wind speed is $0.06 \mathrm{~m}$. Then the blade becomes pitch, and the tip deflection decreases with the increase of wind speed. In the wind speed range of $3-17 \mathrm{~m} / \mathrm{s}$, the linear and nonlinear results agrees well. However, within the wind speed of $18-25 \mathrm{~m} / \mathrm{s}$, the nonlinear results are smaller than the linear ones, and the difference increases with the wind speed increasing. In the process of nonlinear analysis, the enhancement of blade stiffness due to displacement is taken into account. Moreover, the blade tip elastic deflection is larger under high wind speed, and the geometrically nonlinear effect are fully considered in the geometrically exact beam theory, so the elastic deflection of the blade can be predicted more accurately; as for $15 \mathrm{MW}$ wind turbine, the changing trend is same. The linear results show that the maximum tip displacement is $15.1 \mathrm{~m}$ near the rated wind speed. The nonlinear results are in good agreement with the linear results at the wind speed of $3-7 \mathrm{~m} / \mathrm{s}$, but less than the linear results at the wind speed of $8-13 \mathrm{~m} / \mathrm{s}$, and the difference is the largest near the rated wind speed, with a value of $3.4 \mathrm{~m}$. In the range of $14-25 \mathrm{~m} / \mathrm{s}$, the nonlinear results are also smaller than the linear ones, and the difference increases with the increase of wind speed. Its shows that the blade of $15 \mathrm{MW}$ shows strong nonlinearity near the rated wind speed and high wind speed. Since the ordinary modal superposition method has failed, the consideration of geometric nonlinearity is particularly important.

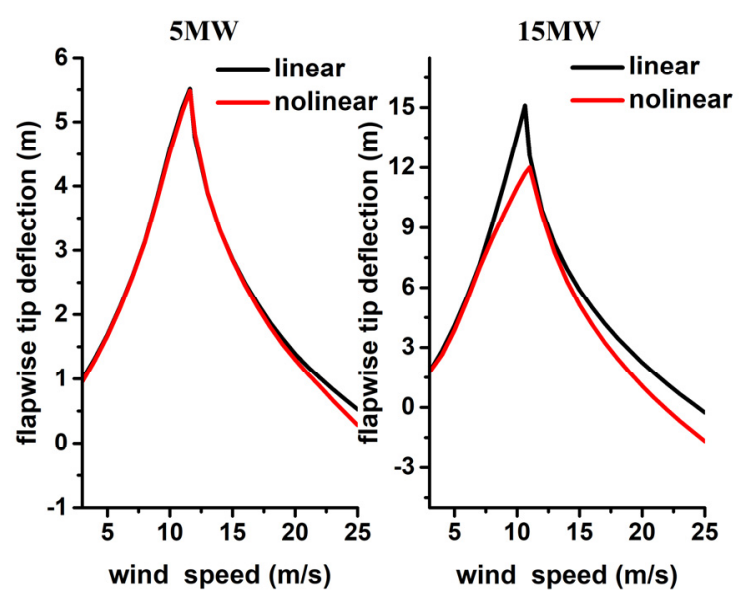

Fig.4 Flapwise tip deflection of wind turbines under different wind speeds

As shown in Fig5, the variation trend of flapwise root moment is the same as that of flapwise tip deflection, which reaches the maximum near the rated wind speed. For the 5MW wind turbine, the linear and nonlinear 
results are in good agreement, and the nonlinear results are sightly smaller than the linear ones at the rated wind speed. As for the $15 \mathrm{MW}$ wind turbine, the nonlinear results are smaller than the linear results in the range of $8-11 \mathrm{~m} / \mathrm{s}$ wind speed, and the difference is larger. Near the rated wind speed, the difference reached the maximum. At other wind speed, it's a good match. The flapwise root moment is mainly determined by the aerodynamic load, the torsional deformation of the blade is closely related to the angle of attack, which affects the aerodynamic load. The modal superposition method based on the simple beam theory only consider the bending degree of freedom of the blades, and has a higher calculation accuracy for the root moment of the $5 \mathrm{MW}$ blade. However, for the blades of high-power wind turbines, failure to take account of the degree of freedom of torsion would result in such a large difference at rated wind speed.

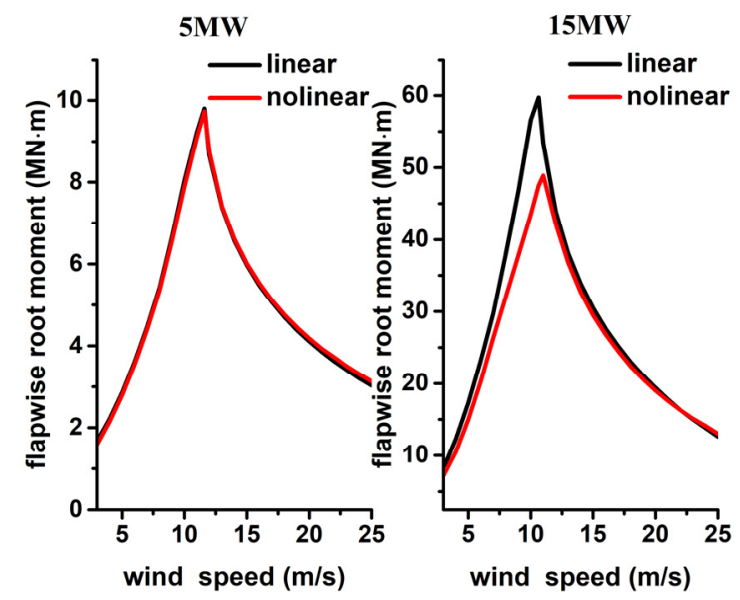

Fig.5 Flapwise root moment of wind turbines under different wind speeds

\section{Summary and Conclusion}

As shown in Fig.6, the percentage difference between the flapwise tip displacement of 5MW and $15 \mathrm{MW}$ wind turbines using linear and nonlinear methods at rated wind speed is presented respectively. Red repensents a $5 \mathrm{MW}$ wind turbine with a blade span of $61.5 \mathrm{~m}$;black repensents a $15 \mathrm{MW}$ wind turbine with a blade span of $117 \mathrm{~m}$. It can be seen that the difference of blade tip displacement of $15 \mathrm{MW}$ wind turbine is almost 29 times that of 5MW wind turbine under two different methods. From the difference, the importance of considering geometric nonlinearity for 100 -meter-scale blades is illustrated.

Therefore, for a 5MW turbine, the modal superposition method based on the simple beam theory is still applicable because the prebend of 5MW wind turbine is small, there is no cross-sectional coupling due to lamination of anisotropic composites. Torsional, stretch and shear efects are almost negligible. Secondly, the centroid offset is very small and will not cause great changes in dynamic reponse. And the lowest wind excitation mode is mainly the bending mode, its displacement is small enough to be accurately captured by the linear term in simple beam theory; as for the 100 - meter-scale blade of high-power wind turbines such as $15 \mathrm{MW}$ wind turbine, it has the characteristics of large flexibility and large deformation, it also has the coupling effect of bending and torsion, the modal superposition method is no longer suitable for this kind of blade, so the aeroelastic method considering geometric nonlinearity must be used to simulate this kind of blade.

After considering the geometric nonlinearity, the results of flapwise tip displacement and root moment are obviously reduced. Therefore, the flexibility or quality of the blades can be further reduced while ensuring the safety factor, thereby reducing the production cost of blade. With the increase of the size of composite blades, the new aeroelastic method considering geometric nonlinearity is necessary when small deformation sssumption is invalid. It must be used to model blades with composite-material-induced couplings and large deflections.

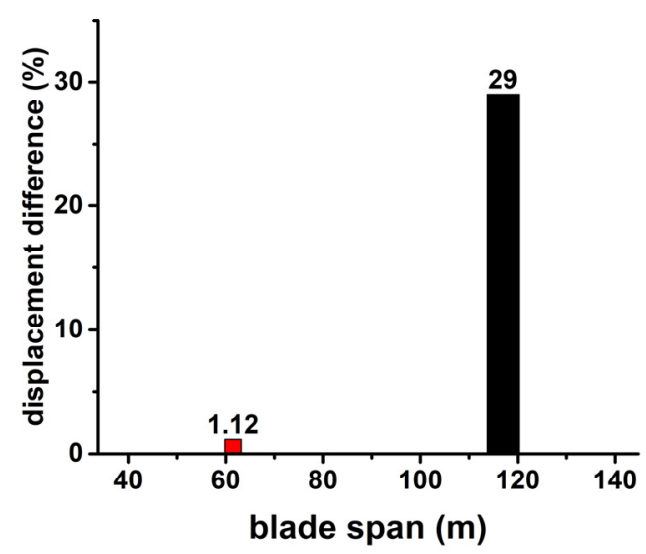

Fig.6 Percentage difference of flapwise tip deflection between linear and nonlinear methods at the rated wind speed

\section{Acknowledgments}

This work was funded jointly by the National Key Research and Development Program of China (No. 2019YFB1503700), and the Priority Academic Program Development of Jiangsu Higher Education Institutions.

\section{References}

1. W. Qi; S. Michael A.; J. Jason; J. Nick; J. Bonnie, Wind Energy, (2017)

2. X. D. Zhou, K. F. Huang, Z. Li, Int. J. Non Linear Mech., 104, (2018)

3. MM. Rezaei, M. Behzad, H. Haddadpour, H. Morad i. Nonlinear Dyn, (2017)

4. X. Qu, Y. Li, Y. Tang, W. chai, Z. Gao. Applied Oc ean Research, 97, (2020)

5. Z. Gao, Y. Li, T. Wang, et al. Appl. Math. Mech-En gl. 42, 4(2021)

6. Z. Gao, Y. Li, T. Wang, et al. Renewable Energy. 1 72(2021) 